\title{
BMJ Open A community-based survey for different abnormal glucose metabolism among pregnant women in a random household study (SAUDI-DM)
}

\author{
Khalid Al-Rubeaan, ${ }^{1}$ Hamad A Al-Manaa, ${ }^{2}$ Tawfik A Khoja, ${ }^{3}$ Amira M Youssef, ${ }^{1}$ \\ Ahmad H Al-Sharqawi, ${ }^{4}$ Khalid Siddiqui, ${ }^{5}$ Najlaa A Ahmad ${ }^{4}$
}

To cite: Al-Rubeaan $\mathrm{K}$, Al-Manaa HA, Khoja TA, et al. A community-based survey for different abnormal glucose metabolism among pregnant women in a random household study (SAUDIDM). BMJ Open 2014;4: e005906. doi:10.1136/ bmjopen-2014-005906

- Prepublication history for this paper is available online To view these files please visit the journal online (http://dx.doi.org/10.1136/ bmjopen-2014-005906).

Received 12 June 2014 Revised 16 July 2014 Accepted 28 July 2014

CrossMark

For numbered affiliations see end of article.

Correspondence to Dr Khalid Al-Rubeaan; krubean@ksu.edu.sa

\section{ABSTRACT}

Objective: To assess the prevalence and risk factors of gestational diabetes mellitus (GDM) in a population known to have a high prevalence of abnormal glucose metabolism.

Methods: A household random population-based cross-sectional study of 13627 women in the childbearing age, who were subjected to fasting plasma glucose if they were not known to have been diagnosed before with any type of diabetes. GDM cases were diagnosed using the International Association of Diabetes and Pregnancy Study Group (IAPSG) criteria.

Results: The overall GDM prevalence was $36.6 \%$, categorised into $32.4 \%$ new cases and $4.2 \%$ known cases. Another $3.6 \%$ had preconception type 1 or 2 diabetes. GDM cases were older and had a significantly higher body mass index, in addition to a higher rate of macrocosmic baby and history of GDM. Monthly income, educational level, living in urban areas and smoking were not found to be significantly different between normal and GDM cases. The most important and significant risk factors for GDM were history of GDM, macrosomic baby, obesity and age $>30$ years. However, hypertension, low high-density lipoprotein, family history of diabetes and increased triglycerides did not show any significant effect on GDM prevalence in this cohort.

Conclusions: This society is facing a real burden of abnormal glucose metabolism during pregnancy, where almost half of the pregnant women are subjected to maternal and neonatal complications. Early screening of pregnant women, especially those at a high risk for GDM, is mandatory to identify and manage those cases.

\section{INTRODUCTION}

Ever since gestational diabetes mellitus (GDM) was first recognised in $1967,{ }^{1}$ it has been the primary focus of interest for clinicians and scientists, due to its increased risk of fetal macrosomia, neonatal hypoglycaemia, jaundice, polycythaemia, hypocalcaemia, and

\section{Strengths and limitations of this study}

- Our study is a community household-based study seeking cases with abnormal glucose metabolism among pregnant women in a country ranked the seventh worldwide in terms of diabetes prevalence.

- The new International Association of Diabetes and Pregnancy Study Group (IAPSG) criteria were used to screen for gestational diabetes mellitus (GDM) cases at a community setup rather than hospital based, especially when there are a limited number of studies that have used these criteria.

- The studied cohort is unique because of its swift socioeconomic transition observed in the Saudi society that gives a good setup to assess different modifiable and non-modifiable risk factors for GDM and to compare this with other ethnicities.

- One of the limitations of this study was the use of fasting plasma glucose (FPG) that was found to have lower sensitivity when compared with the oral glucose tolerance test (OGTT), although it has been recently recommended by the American Diabetes Association (ADA) in 2011 for GDM screening.

also its increased frequency of maternal hypertensive disorders with the need for cesarean deliveries. ${ }^{2}$ GDM, an asymptomatic disorder, is defined as glucose intolerance with the onset or its first recognition during pregnancy. It is the most common metabolic disorder accounting for almost $90 \%$ of all diabetes cases during pregnancy, and has been found to be present in approximately $7 \%$ of all pregnant women. ${ }^{3}$ Moreover, mothers with a history of GDM are at a greater risk of developing type 1 diabetes $(5-10 \%)^{4}$ or type 2 diabetes (over $70 \%),{ }^{5}$ while their offspring are more likely to be obese or to suffer from diabetes in their later life. ${ }^{6}$ The global prevalence of GDM ranges between $1 \%$ and $14 \%$ 
depending on the population studied and the diagnostic tests used. ${ }^{3}$ The prevalence estimates for GDM in 2007 were the following: $11.6 \%$ for Asian Indians, $10 \%$ for Vietnamese, $9.8 \%$ for Pacific Islanders and $7.9 \%$ for East Asians and while it ranged from $4 \%$ to $6 \%$ for Hispanics, it was found to be $4 \%$ in non-Hispanic African-Americans and $4.7 \%$ in non-Hispanic Caucasians. ${ }^{7}$

Even though a hospital-based study among Saudi women using the National Diabetes Data Group (NDDG) criteria $^{8}$ showed the GDM prevalence to be $12.5 \%$, no community-based study has been undertaken to look into the extent of this medical problem in the Kingdom of Saudi Arabia even after knowing that the risk factors of GDM are highly prevalent in this society.

During the childbearing period, women are subjected to increased risk of abnormal glucose metabolism with the progression of age and other general risk factors including obesity and physical inactivity. Several reproductive risk factors, namely parity, the presence of polycystic ovary, the history of GDM, macrosomic baby $>4.5 \mathrm{~kg}$, and the use of contraceptive drugs, have been found to significantly increase the risk of abnormal glucose metabolism in this group of women. ${ }^{9} 10$

Based on the Hyperglycemia and Adverse Pregnancy Outcome (HAPO) study results, the International Association of Diabetes and Pregnancy Study Group (IADPSG) recommended lowering the fasting plasma glucose threshold for the GDM diagnosis to $\geq 92 \mathrm{mg} / \mathrm{dL}$ $(5.1 \mathrm{mmol} / \mathrm{L}),{ }^{11}$ which was also adopted by the American Diabetes Association (ADA) in 2011. ${ }^{12}$ These new diagnostic criteria, as expected, resulted in a significant increase in GDM prevalence in many countries, ${ }^{13-15}$ where it had increased from $10.3 \%$ to $30.1 \%$ in Mexicans ${ }^{15}$ while reaching up to $30.5 \%$ and $37.7 \%$ in Norway and UAE. ${ }^{16} 17$

The aim of the present study was to investigate the prevalence of different abnormal glucose conditions among pregnant women in the Kingdom of Saudi Arabia. The prevalence of GDM and its different risk factors were also investigated using the new IADPSG diagnostic criteria.

\section{METHODS}

The Saudi Abnormal Glucose Metabolism and Diabetes Impact Study (SAUDI-DM study) is a household random population-based cross-sectional study conducted at a national level in the Kingdom of Saudi Arabia during the period 2007-2009. After adjusting for age, gender and geographical distribution according to the 2007 census, a total of 53370 participants were used for the assessment of different states of abnormal glucose metabolism. Of those participants, 13627 participants were found to be women in their childbearing age (1849 years) as shown in figure 1 , and among those women participants, 549 participants were found to be pregnant in different trimesters confirmed by their pregnancy tests, who represented $4 \%$ of the total women participants' cohort. All the SAUDI-DM participants gave their consent to participate and were subjected to interviews. Clinical assessment of the participants, which included pregnant women, where their clinical data including age, family history of diabetes, history of GDM and macrosomic baby, educational level, monthly income, smoking status, and residency area were all collected by specially trained primary care physicians from the 13 official regions. The anthropometric parameters including weight, height, body mass index (BMI), waist-to-hip ratio and blood pressure were all measured by trained nurses.

Pregnant women who were not known to be suffering from diabetes were tested for FPG and lipids profile after overnight fasting for at least $10 \mathrm{~h}$ and 3 days of usual activity and diet. Using the IADPSG criteria,

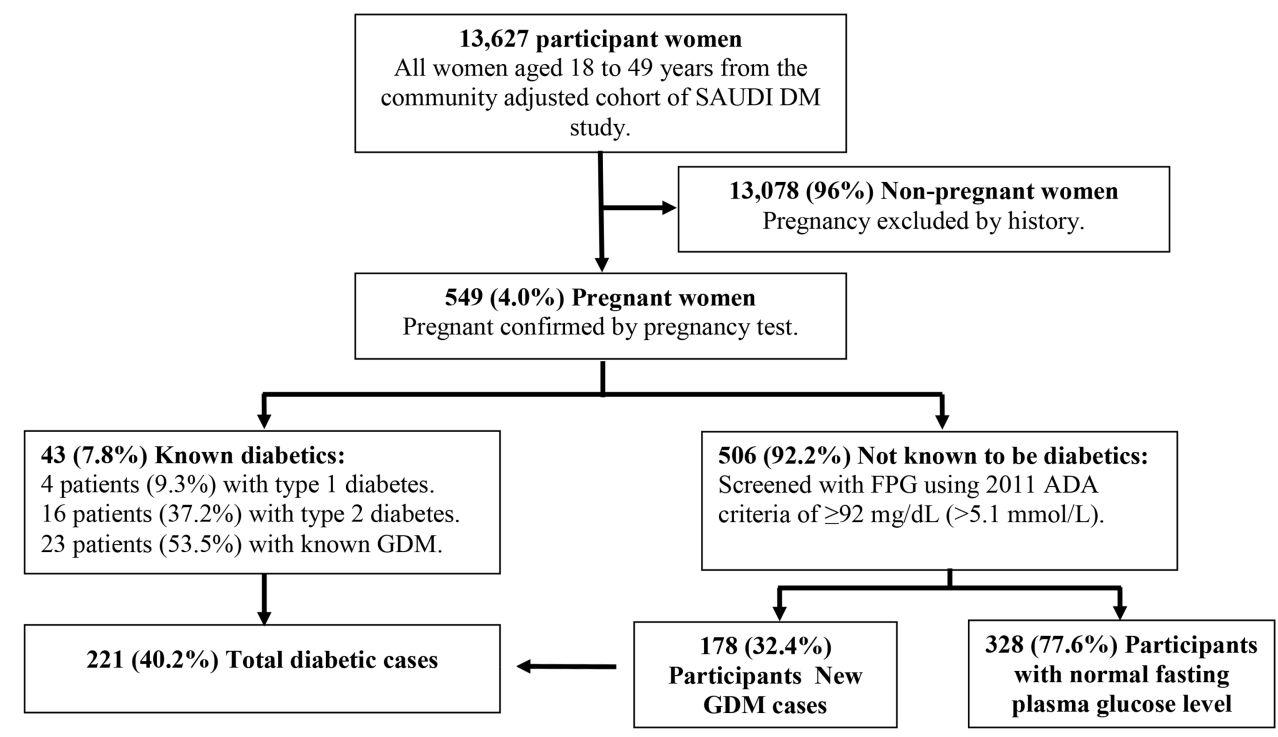

Figure 1 Sample selection of childbearing age pregnant women from of the Saudi Abnormal Glucose Metabolism and Diabetes Impact Study (SAUDI-DM) cohort classified according to abnormal glucose metabolism status. 
pregnant women were diagnosed with GDM if FPG was $\geq 92 \mathrm{mg} / \mathrm{dL}(5.1 \mathrm{mmol} / \mathrm{L})$.

All the blood samples collected using vacuum tubes containing sodium fluoride were transported to the Strategic Center for Diabetes Research laboratory in Riyadh. Blood glucose assessment was performed using the glucose oxidase-peroxidase methodology, serum cholesterol assessment was performed using the cholesterol oxidase-peroxidase methodology, and high-density lipoprotein (HDL), low-density lipoprotein and triglyceride assessments were performed using the direct glycerokinase oxidase-peroxidase methodology as provided by Mindray (B5BS-200) chemistry analyser reagent (China).

\section{Statistical analysis}

All data were analysed using SPSS V.17. Descriptive analyses and frequency tables were performed using this programme for all variables. The $\chi^{2}$ test was used for categorical variables, while the $t$ test was used for continuous variables. OR with $95 \%$ CI were used for assessing the risk factor using univariate analysis. $p$ Value of $<0.05$ was used as the level of significance.

\section{RESULTS}

Table 1 describes the characteristics of the study population. Of the selected cohort containing Saudi pregnant women, 43 participants were women with known diabetes
(7.8\%), of which 4 participants $(9.3 \%)$ were found to be patients with type 1 diabetes, 16 participants (37.2\%) were found to be patients with type 2 diabetes, and 23 participants $(53.5 \%)$ were found to be with GDM. And among the remaining 506 women not known to be suffering from diabetes, $178(32.4 \%)$ were found to be new GDM cases. The studied cohort was divided into three age groups: the first group is between 18 and 29 years of age with a total number of $264(48.1 \%)$. The second age group is between 30 and 39 years, totalling to 212 $(38.6 \%)$, while the third group is between 40 and 49 years with a total number of $73(13.3 \%)$.

When comparing the clinical characteristics of GDM cases with the clinical characteristics of pregnant women without diabetes, the GDM cases were found to be significantly older $(33.26 \pm 7.63$ years $)(p<0.0001)$ and more obese as presented by their body weight (73.94 $\pm 14.83 \mathrm{~kg})$ and BMI $\left(30.27 \pm 5.57 \mathrm{~kg} / \mathrm{m}^{2}\right) \quad(\mathrm{p}<0.0001)$. There was no significant difference between the two groups in their mean height and waist-to-hip ratio $(\mathrm{p}=0.176)$. The GDM cases had a significantly higher mean systolic blood pressure (SBP) at 114.02 $\pm 11.61 \mathrm{~mm} \mathrm{Hg}(\mathrm{p}=0.002)$ and a low diastolic blood pressure at $72.71 \pm 8.81 \mathrm{~mm} \mathrm{Hg}(\mathrm{p}=0.160)$. And as expected, the mean FPG was found to be significantly higher in GDM cases at $6.55( \pm 2.21) \mathrm{mmol} / \mathrm{L}$ when compared with the mean FPG of the normal women at 4.21 $\pm 0.65 \mathrm{mmol} / \mathrm{L}(\mathrm{p}<0.0001)$. Lipids showed no significant

Table 1 Baseline clinical and biochemical characteristics of the selected cohort, newly diagnosed gestational diabetes mellitus (GDM) and normoglycaemic pregnant participants

\begin{tabular}{|c|c|c|c|}
\hline & Normal $(n=328)$ & GDM $(n=201)$ & p Value* \\
\hline Mean participant age in years $( \pm S D)$ & $29.63( \pm 7.53)$ & $33.26( \pm 7.72)$ & $<0.0001$ \\
\hline Mean weight in $\mathrm{kg}( \pm \mathrm{SD})$ & $67.11( \pm 13.55)$ & $73.94( \pm 14.83)$ & $<0.0001$ \\
\hline Mean height in $\mathrm{cm}( \pm \mathrm{SD})$ & $154.98( \pm 5.42)$ & $156.37( \pm 6.97)$ & 0.057 \\
\hline Mean $\mathrm{BMI}=\mathrm{kg} / \mathrm{m}^{2}( \pm \mathrm{SD})$ & $27.97( \pm 5.37)$ & $30.27( \pm 5.57)$ & $<0.0001$ \\
\hline Mean waist/hip ratio $( \pm S D)$ & $0.89( \pm 0.14)$ & $0.87( \pm 0.14)$ & 0.176 \\
\hline Mean systolic blood pressure, $\mathrm{mm} \mathrm{Hg}( \pm \mathrm{SD})$ & $110.03( \pm 10.84)$ & $114.02( \pm 11.61)$ & 0.002 \\
\hline Mean diastolic blood pressure, $\mathrm{mm} \mathrm{Hg}( \pm \mathrm{SD})$ & $71.34( \pm 8.32)$ & $72.71( \pm 8.81)$ & 0.160 \\
\hline Mean fasting plasma glucose, $\mathrm{mmol} / \mathrm{L}( \pm S D)$ & $4.21( \pm 0.65)$ & $6.55( \pm 2.21)$ & $<0.0001$ \\
\hline Mean total cholesterol, $\mathrm{mmol} / \mathrm{L}( \pm \mathrm{SD})$ & $5.58( \pm 1.29)$ & $5.41( \pm 1.32)$ & 0.278 \\
\hline Mean HDL cholesterol, $\mathrm{mmol} / \mathrm{L}( \pm \mathrm{SD})$ & $1.15( \pm 0.32)$ & $1.07( \pm 0.32)$ & 0.025 \\
\hline Mean LDL cholesterol, mmol/L ( \pm SD) & $3.57( \pm 1.05)$ & $3.52( \pm 1.16)$ & 0.704 \\
\hline Mean triglycerides, $\mathrm{mmol} / \mathrm{L}( \pm \mathrm{SD})$ & $1.81( \pm 0.94)$ & $1.84( \pm 0.95)$ & 0.778 \\
\hline Number with family history of diabetes (\%) & $157(47.87)$ & $103(51.24)$ & 0.451 \\
\hline Number with history of gestational diabetes (\%) & $12(3.66)$ & 26 (12.94) & $<0.0001$ \\
\hline Number with history of macrosomia (\%) & $12(3.66)$ & $23(11.44)$ & $<0.0001$ \\
\hline Number of illiterate participants (\%) & $74(22.56)$ & $35(17.41)$ & 0.155 \\
\hline Number with less than high school education (\%) & $113(34.45)$ & $78(38.81)$ & 0.311 \\
\hline Number with more than high school education (\%) & 141 (42.99) & $88(43.78)$ & 0.858 \\
\hline Number with monthly income <4000 SR $†$ (\%) & $153(46.65)$ & 73 (36.32) & 0.020 \\
\hline Number with monthly income $4000-8000 \mathrm{SR} \dagger(\%)$ & $97(29.67)$ & 69 (34.33) & 0.253 \\
\hline Number with monthly income >8000 SR $†(\%)$ & $78(23.78)$ & 59 (29.35) & 0.156 \\
\hline Number of smoking participants (\%) & $4(1.22)$ & $2(1.00)$ & 0.811 \\
\hline Number of participants living in urban areas $\ddagger$ (\%) & $213(64.94)$ & $135(67.16)$ & 0.601 \\
\hline
\end{tabular}

${ }^{*} p$ Value was calculated as difference between normal and GDM cases.

†Saudi Riyal (SR) is equivalent to US\$0.267.

$\ddagger$ According to the definition given by the Ministry of Municipal and Rural Affairs. 


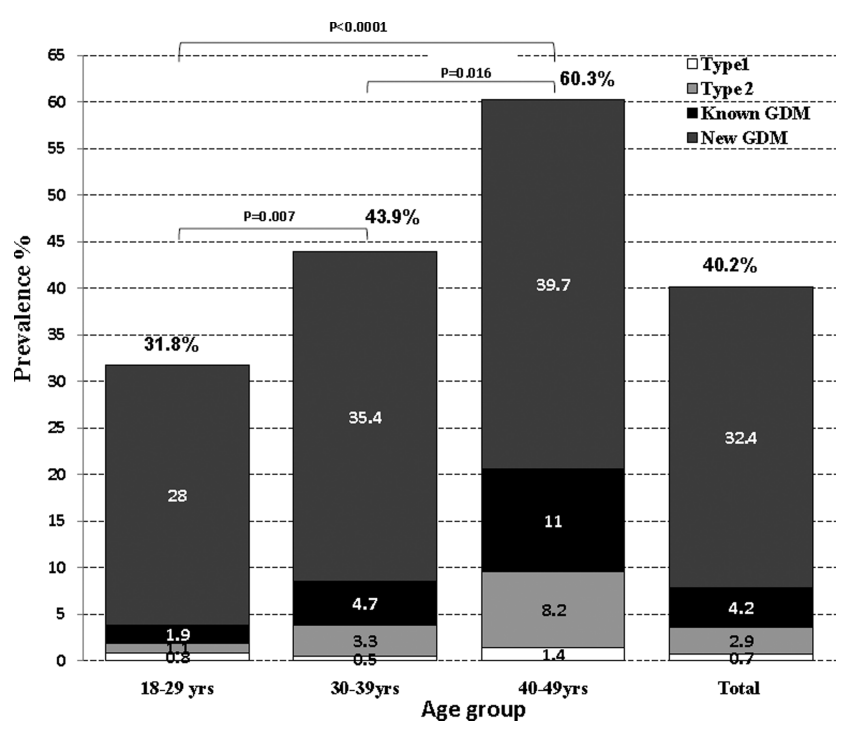

Figure 2 The prevalence of abnormal glucose metabolism in pregnant Saudi women aged 18-49 years.

difference between the two groups with the exception of mean HDL, which was found to be lower in the GDM cases at $1.07 \pm 0.32 \mathrm{mmol} / \mathrm{L}$ when compared with the mean HDL for normal women at $1.15 \pm 0.32 \mathrm{mmol} / \mathrm{L}$ $(\mathrm{p}=0.025)$. The percentage of women with positive family history of diabetes was not found to be significantly different between the GDM cases and normal women ( $p=0.451$ ), while it was found to be significantly higher for the history of GDM and macrocosmic baby $>4.5 \mathrm{~kg}$ among GDM cases at $12.49 \%$ vs $3.66 \%$ $(\mathrm{p}<0.0001)$ and $11.44 \%$ vs $3.66 \%(\mathrm{p}<0.0001)$, respectively, when compared with normal women. Educational level showed no significant difference in percentage between the two groups, while the percentage of women with monthly income $<4000$ SR was found to be significantly higher among normal women $(p=0.02)$. Smoking or living in urban areas also showed no significant difference between the two groups $(\mathrm{p}=0.811$ and $\mathrm{p}=0.601)$.

Figure 2 demonstrates the prevalence of different types of abnormal glucose metabolism in the selected cohort of Saudi pregnant women aged 18-49 years. Type 1 diabetes was found in $0.7 \%$ of the total sample, while type 2 was reported in $2.9 \%$ of the total sample. $4.2 \%$ of the total sample were known cases of GDM and $32.4 \%$ of the total sample were newly discovered GDM cases. The overall prevalence of abnormal glucose metabolism showed an age specific increase with a significant difference from $31.8 \%$ to $43.9 \%$ and $60.3 \%$ in the age groups $18-29,30-39$ and $40-49$ years respectively. The prevalence of newly discovered GDM cases showed an increase from $28.0 \%$ to $35.4 \%$ and $39.7 \%$ in the three groups, respectively. This was also observed in the prevalence of known GDM cases from $1.9 \%$ in the age group $18-29$ years to $4.7 \%$ in the age group 30-39 years and peaking at $11 \%$ in the age group $40-49$ years. The prevalence of known patients with type 1 and 2 diabetes was $0.8 \%$ and $1.1 \%$ for the age group $18-29$ years, $0.5 \%$ and $3.3 \%$ for the age group $30-39$ years and $1.4 \%$ and $8.2 \%$ for the age group 40-49 years. The ratio of known to unknown GDM cases was calculated to be1:15, 1:8 and 1:4 for the age groups 18-29, 30-39 and 40-49 years, respectively.

Figure 3 shows the OR (95\% CI), where the history of GDM was found to be a highly significant risk factor with OR 3.91 (95\% CI) (1.93 to 7.95) $(\mathrm{p}<0.0001)$. It was followed by the history of macrosomic baby weighing $>4.5 \mathrm{~kg}$ that had OR $(95 \% \mathrm{CI})$ of 3.40 (1.65 to 7.00) $(\mathrm{p}<0.0001)$. Obesity was the third significant risk factor with OR (95\% CI) 2.50 (1.35 to 4.60) followed by age $>30$ years, where the OR $(95 \% \mathrm{CI})$ was 2.00 (1.26 to $3.18)$, both with a significant $p$ value $(p=0.003)$. Other risk factors, which include hypertension, low HDL, and family history of diabetes, showed a non-significant increased risk for GDM presented by OR $(95 \%$ CI) (1.50 (0.47 to 4.75$), 1.34$ (0.76 to 2.36$)$, and 1.15 (0.81 to 1.63)), respectively. Lastly, the high triglycerides showed no significant risk for GDM.

\section{DISCUSSION}

This study investigated the occurrence of abnormal glucose metabolism during pregnancy using a community-based rather than a traditional hospital based setup. A study with this setup provides a better image of the extent of this medical problem, especially when using the new diagnostic criteria to identify GDM cases at a community level. This study shows the importance of
Figure 3 OR for gestational diabetes mellitus risk factors among Saudi pregnant participants.

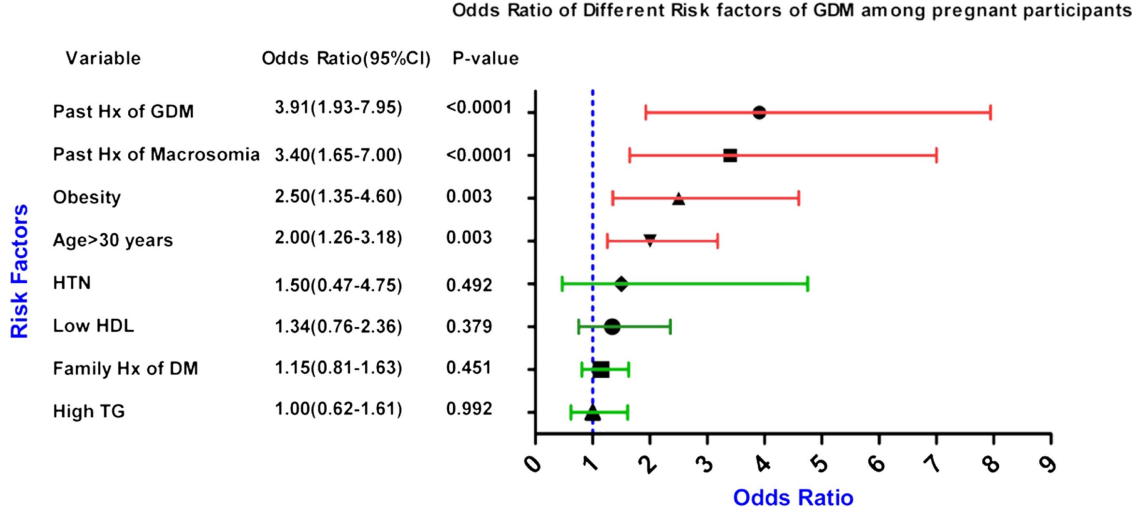


subjecting women to GDM screening, particularly when it is known that only $4.2 \%$ of pregnant women were picked up by the health system (known GDM cases). The current survey picked up another $32.4 \%$ of GDM cases; this would mean that only one of every nine GDM cases was detected by the antenatal screening, which also clearly demonstrates that there are many undiagnosed GDM cases at the community level. Another crucial finding of this study was that the overall GDM prevalence was $36.6 \%$, threefold higher than what was reported earlier, ${ }^{8}$ but similar to the findings of studies from other ethnicities using these criteria, ${ }^{15}$ which is most likely due to the lower cut-off value of the new criteria used and the high prevalence of overweight and obesity reflected by a high mean BMI in the studied cohort. This may be coupled with the fact that such a community-based study would identify more GDM cases, especially in a society where diabetes prevalence is found to be increasing over the past three decades. ${ }^{18}$ The patients with pre-existing types 1 and 2 diabetes in this study represent $3.6 \%$ of all pregnancies, which was three times more than what had been reported in North America ${ }^{19}$ and is most likely a reflection of the high prevalence of abnormal glucose metabolism in our population.

This studied cohort showed that the mean age for GDM Saudi pregnant women was higher than what had been earlier reported in other studies involving the Chinese and Indian populations, ${ }^{20}{ }^{21}$ which could be the result of cultural factors that encourage women to get pregnant even at an older age, in addition to high parity. ${ }^{22}$ This may also have contributed to the higher prevalence of GDM in this cohort with the mean age of GDM cases found to be significantly higher than the normal cases. Another explanation for the higher prevalence of GDM in our cohort is provided by the presence of high mean BMI when compared with the studies involving Caucasian and Asian populations. ${ }^{23}{ }^{24}$ More than $50 \%$ of GDM cases had a family history of diabetes that was not significantly different from normal cases and this could be explained by the high prevalence of diabetes and high consanguinity rates in our society. ${ }^{25}$ As expected and seen from other studies, ${ }^{21}{ }^{26}$ the history of GDM and macrosomic baby was found to be higher in GDM cases than in normal pregnant women, where it was almost four times higher in our GDM cohort. Neither education nor monthly income showed any significant increase in GDM cases, with the exception of monthly income less than 4000 SR that was found to be associated with a decreased percentage of GDM cases. Surprisingly, both smoking and living in urban areas showed no significant difference between the two groups, which could be evidently explained on the basis of cultural effects, where smoking is not culturally acceptable for women in Saudi Arabia, and on the basis of high socioeconomic status that has eliminated the difference between urban and rural areas in the Kingdom.

Consistent with many studies ${ }^{27}$ and as expected, the prevalence of preconception diabetes, known GDM and newly discovered GDM cases showed a significant increase with age, peaking at age more than 40 years. This could be explained by the effect of age progression and muliparity in our society. ${ }^{23}$ The ratio of the previously known to newly discovered GDM cases decreased with the progression of age, which could be explained by the fact that older women were subjected to screening more than younger women as also observed by other researchers. ${ }^{27}$

This study also shows that, history of GDM increased the risk for GDM significantly, which was found to be much lower than what had been observed in the studies involving Persian, Indian and Caucasian populations, ${ }^{21} 26$ which could be an effect of the nature of the study sample, with our sample being community based and the rest being hospital based. History of macrosomia was the second risk factor found to increase GDM cases in the Saudi pregnant women, close to what had been reported in other ethnicities. ${ }^{21} 28$ Obesity and being more than 30 years of age, known to be important risk factors for diabetes in general, significantly increased the risk for GDM, as reported earlier in many ethnicities. ${ }^{20}{ }^{23}$ Hypertension, low HDL, family history of diabetes and high triglycerides were all found to be non-significant risk factors for GDM in this study. Hypertension and low HDL increased the risk for developing GDM in this population but not to a significant level similar to the Caucasians, ${ }^{23}$ while positive family history of diabetes had a minimal non-significant increase, which could have resulted from high consanguinity rates and family history of diabetes in the general population. ${ }^{25}$ Although high triglyceride was known to be a risk factor for GDM, ${ }^{23}$ this study showed no effect of this risk factor, which could be due to the high mean triglycerides in normal and GDM cases.

This study derives strength from being a communitybased household survey representing a normal distribution cohort that was randomly selected. One of the limitations of this study was the use of FPG which was found to have lower sensitivity when compared with the oral glucose tolerance test, ${ }^{29}$ although it has been recently recommended by the ADA for GDM screening. ${ }^{12}$ Another limitation of this study was the exclusion of pregnancy by history alone, which may have excluded some pregnant women in their early pregnancy in addition to the lack of obstetrical and diet history. Although this study involved special ethnicity, the results could be generalised at an international level, especially when looking at risk factors.

\section{CONCLUSIONS}

Around $40 \%$ of Saudi pregnant women suffer from either pre-existing diabetes or GDM secondary to the increased prevalence of its risk factors in this society. A limited number of GDM cases are picked up by the health system indicating the weak antenatal screening and thereby warranting an extensive public and medical 
staff education. The GDM risk factors that are significant in this society include: history of GDM and macrosomic baby, obesity and age more than 30 years that should be considered for early screening of pregnant women. Public awareness programmes for reducing modifiable risk factors like obesity would contribute to decreasing the prevalence of GDM and prenatal mortality.

\section{Author affiliations}

${ }^{1}$ University Diabetes Center, College of Medicine, King Saud University, Riyadh, Saudi Arabia

${ }^{2}$ Ministry of Health, Riyadh, Saudi Arabia

${ }^{3}$ Gulf Corporation Council, Riyadh, Saudi Arabia

${ }^{4}$ Biostatistics Department, Strategic Center for Diabetes Research, King Saud University, Riyadh, Saudi Arabia

${ }^{5}$ Biochemistry Department, Strategic Center for Diabetes Research, King Saud University, Riyadh, Saudi Arabia

Acknowledgements The authors would like to acknowledge the staff at the University Diabetes Center and the staff of the primary care centers from the Ministry of Health for their collaboration in conducting the study.

Contributors All the authors have contributed towards the conduct of the study and preparation of the manuscript. KAR designed the study, wrote the manuscript, designed figures and interpreted data, and approved the final version to be published. HAAM supervised the field work in different health sectors in relation to this project. TAK provided training to the research physicians and did the statistical analysis. AMY researched data, wrote the manuscript, and critically revised the article. AHAS analysed data, developed figures, interpreted data and approved the final version to be published. KS handled and analysed blood samples, revised the article critically and approved the final version to be published. NAA researched data, analysed data and approved the final version to be published.

Funding This study was funded by the University Diabetes Center at King Saud University, Ministry of Health and the Tawuniya Company for health insurance.

Competing interests None.

Ethics approval This study has been approved by the Institutional Review Board (IRB) at the College of Medicine, King Saud University.

Provenance and peer review Not commissioned; externally peer reviewed.

Data sharing statement No additional data are available.

Open Access This is an Open Access article distributed in accordance with the Creative Commons Attribution Non Commercial (CC BY-NC 4.0) license, which permits others to distribute, remix, adapt, build upon this work noncommercially, and license their derivative works on different terms, provided the original work is properly cited and the use is non-commercial. See: http:// creativecommons.org/licenses/by-nc/4.0/

\section{REFERENCES}

1. O'Sullivan JB, Mahan CM. Criteria for the oral glucose tolerance test in pregnancy. Diabetes 1964;13:278-85.

2. American Diabetes Association. Gestational diabetes mellitus. Diabetes Care 2004;27(Suppl 1):S88-90.

3. Setji TL, Brown AJ, Feinglos MN. Gestational diabetes mellitus. Clin Diabetes 2005;23:17-24.

4. Damm P, Kuhl C, Buschard K, et al. Prevalence and predictive value of islet cell antibodies and insulin autoantibodies in women with gestational diabetes. Diabet Med 1994;11:558-63.

5. Kim C, Newton KM, Knopp RH. Gestational diabetes and the incidence of type 2 diabetes: a systematic review. Diabetes Care 2002;25:1862-8.
6. Buchanan TA, Xiang AH, Page KA. Gestational diabetes mellitus: risks and management during and after pregnancy. Nat Rev Endocrinol 2012;8:639-49.

7. Kim SY, England L, Sappenfield W, et al. Racial/ethnic differences in the percentage of gestational diabetes mellitus cases attributable to overweight and obesity, Florida, 2004-2007. Prev Chronic Dis 2012;9:E88.

8. Ardawi MS, Nasrat HA, Jamal HS, et al. Screening for gestational diabetes mellitus in pregnant females. Saudi Med J 2000;21:155-60.

9. Ben-Haroush A, Yogev Y, Hod H. Epidemiology of gestational diabetes mellitus and its association with Type 2 diabetes. Diabet Med 2004;21:103-13.

10. Hedderson MM, Ferrara A, Williams MA, et al. Androgenicity of progestins in hormonal contraceptives and the risk of gestational diabetes mellitus. Diabetes Care 2007;30:1062-8.

11. Metzger BE, Lowe LP, Dyer AR, et al:; The HAPO Study Cooperative Research Group. Hyperglycemia and adverse pregnancy outcomes. N Engl J Med 2008;358:1991-2002.

12. American Diabetes Association. Diagnosis and classification of diabetes mellitus. Diabetes Care 2011;34:S62-9.

13. Wei YM, Yang HX. Comparison of the diagnostic criteria for gestational diabetes mellitus in china. Zonghua Fu chan $\mathrm{Ke} \mathrm{Za} \mathrm{Zi}$ 2011;46:578-91.

14. Moses RG, Morris GJ, Petocz P, et al. The impact of potential new diagnostic criteria on the prevalence of gestational diabetes mellitus in Australia. Med J Aust 2011;194:338-40.

15. Reyes-Muñoz E, Parra A, Castillo-Mora A, et al. Effect of the new diagnostic criteria of the International Association of Diabetes and Pregnancy Study groups on the prevelance of gestational diabetes mellitus in urban Mexican women: a cross sectional Study. Endocr Pract 2012;18:146-51.

16. Jenum AK, Mørkrid K, Sletner L, et al. Impact of ethnicity on gestational diabetes identified with the WHO and the modified International Association of Diabetes and Pregnancy Study Groups criteria: a population-based cohort study. Eur J Endocrinol 2012;166:317-24.

17. Agarwal MM, Dhatt GS, Shah SM. Gestational Diabetes Mellitus Simplifying the International Association of Diabetes and Pregnancy diagnostic algorithm using fasting plasma glucose. Diabetes Care 2010;33:2018-20.

18. Al-Nozha MM, Al-Maatouq MA, Al-Mazrou YY, et al. Diabetes mellitus in Saudi Arabia. Saudi Med J 2004:25:1603-10.

19. Lawrence JM, Contreras R, Chen W, et al. Trends in the prevalence of preexisting diabetes and gestational diabetes mellitus among a racially/ethnically diverse population of pregnant women, 19992005. Diabetes Care 2008;31:899-904.

20. Yang H, Wei Y, Gao X, et al. Risk factors for gestational diabetes mellitus in Chinese women: a prospective study of 16286 pregnant women in China. Diabet Med 2009;26:1099-104.

21. Soheilykhah S, Mogibian M, Rahimi-Saghand S, et al. Incidence of gestational diabetes in pregnant women. Iran $J$ Reprod Med 2010;8:24-8.

22. Al Rowaily MA, Alsalem FA, Abolfotouh MA. Cesarean section in a high-parity community in Saudi Arabia: clinical indications and obstetric outcomes. BMC Pregnancy Childbirth 2014;14:92.

23. Gunderson EP, Quesenberry CP Jr, Jacobs DR Jr, et al. Longitudinal study of prepregnancy cardiometabolic risk factors and subsequent risk of gestational diabetes mellitus. Am J Epidemiol 2010;172:1131-43.

24. Cheung NW, Wasmer G, Al-Ali J. Risk factors for gestational diabetes among Asian women. Diabetes Care 2001;24:955-6.

25. Al-Gazali L, Hamamy H, Al-Arrayad S. Genetic disorders in the Arab world. BMJ 2006;333:831-4.

26. Bhat M, Ramesha KN, Sarma SP, et al. Determinants of gestational diabetes mellitus: a case control study in a district tertiary care hospital in south India. Int J Diabetes Dev Ctries 2010;30: 91-6.

27. Makgoba M, Savvidou MD, Steer PJ. An analysis of the interrelationship between maternal age, body mass index and racial origin in the development of gestational diabetes mellitus. BJOG 2012;119:276-82.

28. Cypryk K, Szymczak W, Czupryniak L, et al. Gestational diabetes mellitus-an analysis of risk factors. Endokrynol Pol 2008;59:393-7.

29. Taylor R, Zimmet P. Limitation of fasting plasma glucose for the diagnosis of diabetes mellitus. Diabetes Care 1981;4:556-8. 